\title{
Proper Actions of Certain Nilpotent Affine Groups with Codimension One Orbits
}

\author{
S. Nasrin* \\ Department of Mathematics, University of Dhaka, Dhaka-1000, Bangladesh \\ Received 25 June 2011, accepted in final revised form 4 February 2012
}

\begin{abstract}
Criterion for proper actions has been established for a homogeneous space of reductive type by Kobayashi (Math. Ann. 1989, 1996). On the other hand, an analogous criterion to Kobayashi's equivalent conditions was proposed by Lipsman (1995) for a nilpotent Lie group $G$. Lipsman's Conjecture: Let $G$ be a simply connected nilpotent Lie group. Then the following two conditions on connected subgroups $H$ and $L$ are equivalent: (i) the action of $L$ on $G / H$ is proper; (ii) $L \cap g \mathrm{Hg}^{-1}$ is compact for any $g \in G$. The condition (i) is important in the study of discontinuous groups for the homogeneous space $G / H$, while the second condition (ii) can easily be checked. The implication (i) $\Rightarrow$ (ii) is obvious, and the opposite implication (ii) $\Rightarrow$ (i) was known only in some lower dimensional cases. In this paper we prove the equivalence (i) $\Leftrightarrow$ (ii) for certain affine nilpotent Lie groups $G$.
\end{abstract}

Keywords: Affine nilpotent groups; Homogeneous manifolds; Proper actions; Properly discontinuous actions; Simply connected nilpotent Lie groups; Compact isotropy property (CI); Eigenvalues.

(C) 2012 JSR Publications. ISSN: 2070-0237 (Print); 2070-0245 (Online). All rights reserved. doi: http://dx.doi.org/10.3329/jsr.v4i2.7889 J. Sci. Res. 4 (2), 315-326 (2012)

\section{Introduction}

Let $G$ be a Lie group, $H$ a closed subgroup and $\Gamma$ a torsionless discrete subgroup of $G$. If $H$ is compact, then the double coset space $\Gamma \backslash G / H$ is always Hausdorff in the quotient topology, and equips with a naturally manifold structure such that the map

$$
G / H \rightarrow \Gamma \backslash G / H
$$

is a local diffeomorphism. Such a double coset manifold is called a Clifford-Klein form of the homogeneous manifold $G / H$. A typical example is a closed Riemann surface $M_{g}$ with genus $g \geq 2$, which is a Clifford-Klein form of the Poincaré disk $\operatorname{PSL}(2 ; \mathbb{R}) / S O(2)$ as a special case of the uniformization theorem due to Klein-Koebe-Poincaré.

On the other hand, if $H$ is non-compact then the left action of $\Gamma$ on the homogeneous manifold $G / H$ is not always properly discontinuous and the double coset space $\Gamma \backslash G / H$ is

*E-mail: salma@univdhaka.edu 
not always Hausdorff. A notorious phenomenon, so called "the Calabi-Markus phenomenon" shows that some homogeneous space $G / H$ admits no infinite discrete subgroup $\Gamma$ such that $\Gamma$ acts properly discontinuously on $G / H$. Loosely speaking, this phenomenon indicates that the fundamental group of a manifold $M$ might be not very complicated if $M$ is locally isomorphic to such a homogeneous manifold $G / H$. The first example for the Calabi-Markus phenomenon was found by Calabi and Markus in 1962 [3] for $(G, H)=(O(n, 1), O(n-1,1))$, and generalized by Wolf [22] and Kulkarni [15] for $(G, H)=(O(p, q), O(p ; q-1))$ from 1960s to the early 1980s, and then completely settled by Kobayashi [7] in 1989 for reductive Lie groups $(G, H)$ in terms of real rank conditions. The key lemma of Kobayashi's paper [7] is to establish the criterion of proper actions of continuous subgroups. Here the notion of proper action was proposed by Palais [19] in 1961to single out a nice category of actions of non-compact groups.

In another language, if $G$ is algebraic, one can replace $\Gamma$ by its Zariski- closure $L=\bar{\Gamma}$ and can consider analogous setting concerning $L$. In a basic paper [8], Kobayashi has done exactly that and he has discovered strong results about Clifford-Klein forms of homogeneous spaces $X=G / H$. He then initiates an intense investigation of this. He begins by noting the standard criteria for $\Gamma \backslash X$ to be a manifold - namely if and only if $\Gamma$ acts both properly discontinuously and freely on $X$. He then develops continuous analogs of these properties for $L$-one of them well-known, another less so. We note that the action of $L$ on $X$ is properly discontinuous if and only if the action of $L$ on $X$ is proper and $L$ is discrete, because a discrete and compact set is finite. We will state these properties in the next section.

Since Kobayashi's paper [7] in 1989, there have been extensive studies of CliffordKlein forms of homogeneous spaces $G / H$ for a reductive Lie group $G$ by various methods (see surveys $[10,11]$ and references therein). On the other hand, much has not been studied in the case where $G$ is a nilpotent Lie group, which is supposed to be an opposite extremal case of reductive groups. Lipsman, inspired by the reductive results in ref. [8], proposed the following conjecture for nilpotent groups:

Conjecture 1.1 (Conjecture 4.1(b) of ref. [16]). Let $G$ be a simply connected nilpotent Lie group, and $H, L$ be connected subgroups of $G$. Then $L$ acts properly on $G / H$ if and only if the triplet $(G, H, L)$ has the (CI) property.

Here we say the triplet $(G, H, L)$ has the (CI) property if $L \cap g H g^{-1}=\{e\}$ for any $g \in G$.

This conjecture is built on the simplest case where $G$ is the group of unipotent upper triangular real matrices of order $n=3$ (Kobayashi [8] '92), and is proved also for $n=4$ (Lipsman [16] '95) and remains open for $n \geq 5$. In the case $n=4$ the most difficult case in [16] was the case where $\operatorname{dim} L=\operatorname{dim} G / H-1$, where there is a large room for deformation of $L$ with deformation being parameterized by $2 \times 2$ matrices.

The present paper generalizes this specific case to higher dimension. The formulation is roughly as follows:

$\mathrm{G}=$ upper triangular matrices of order $n=k+2$, 
$\mathrm{H}=$ the isotropy group in $\mathrm{G}$ of the coordinate vector $(0, \ldots, 0,1) \in \mathbb{R}^{k+2}$.

$L \equiv L(A)=k$-dimensional abelian subgroup of $G$, with deformation parameter $A \in M(k, \mathbb{R})$.

As $G / H \cong \mathbb{R}^{k+1}$, our object of study is a proper action of the abelian group $\mathbb{R}^{k}$ on $\mathbb{R}^{k+1}$. Then we shall obtain an explicit criterion on the deformation parameter $A \in$ $M(k, \mathbb{R})$ such that the action of $L(A)$ on $G / H$ is proper. The main result is roughly as follows:

Theorem 1.2 (see Theorem 4.1 for precise statement)

The following three conditions on $A \in M(k, \mathbb{R})$ are equivalent:

(i) the group $L(A)$ acts on $G / H$ properly

(ii) the triplet $(G, H, L(A))$ has the $(\mathrm{CI})$ property

(iii) any eigenvalue of $\mathrm{A}$ is contained in $\{Z \in \mathbb{C}: \operatorname{Im} z \neq 0\} \cup\{0\}$.

In particular, this result supports the conjecture of Lipsman in this special setting. Most part of this paper is devoted to the proof of Theorem 1.2 after making a precise formulation.

The author [17] showed earlier that the conjecture holds when $G$ is two-step nilpotent. Yoshino [24] and Baklouti and Khlif [2] independently proved that it is also the case when $G$ is three-step nilpotent. On the other hand, Yoshino [23] gave a counterexample to this conjecture when $G$ is four-step nilpotent.

This paper is an outcome of the master thesis at the University of Tokyo of the auther. In the meantime, the subject has been rapidly developing. For the convenience to the reader we refer the following very recent papers (see also the references therein), where Baklouti--Khlif [1] treats deformation for discontinuous group for nilpotent groups, Kobayashi [14] for his general program in this area as the pioneer of the field, Okuda [18] for the classification of proper actions of surface groups and $\operatorname{SL}(2, \mathbb{R})$ on semisimple symmetric spaces, and Kassel--Kobayashi [6] for a new spectral theory of the ultrahyperbolic Laplacian under the deformation of discontinuous groups.

\section{Preliminary Results}

In this section we shall recall the basic properties of the action of a Lie group $L$ on $G / H$ or, of the action of a discrete subgroup $\Gamma$ on $G / H$. Basic references for this section are [8, $10,11]$.

\subsection{Proper actions - as a continuous analogue of properly discontinuous actions}


In general, the study of the action of a discrete group is quite difficult. The approach taken in [4] is to approximate the action of discrete groups by that of connected Lie groups. For this purpose, it is crucial to find a continuous analogue of a properly discontinuous action:

Definition 2.1. (see [19]) Suppose that a locally compact topological group $L$ acts continuously on a locally compact topological space $X$. For a subset $\mathrm{S}$ of $X$, we define a subset of $L$ by $\left\{L_{S}=\gamma \in L: \gamma S \cap s \neq \emptyset\right\}$.

The action of $L$ on $X$ is said to be proper if and only if $L_{S}$ is compact for every compact subset $S$ of X.

Recall that the action of $L$ on $X$ is properly discontinuous if and only if the action of $L$ on $X$ is proper and $L$ is discrete, because a discrete and compact set is finite.

\subsection{An observation}

The following elementary observation is a bridge between the action of a discrete group and that of a connected group.

Observation 2.2. ([2], Lemma 2.3) Suppose a Lie group $L$ acts on a locally compact space X. Let $\Gamma$ be a co-compact discrete subgroup of $L$. Then

(1) The $L$-action on $X$ is proper if and only if the $\Gamma$-action is properly discontinuous.

(2) $L \backslash X$ is compact if and only if $\Gamma \backslash X$ is compact.

\subsection{Problems in a continuous analogue}

In view of observation 2.2, Kobayashi [7] posed the following analogous problems in a continuous setting.

Problem 2.3. ([8]) Let $G$ be a Lie group and $H$ and $L$ be closed subgroups.

(1) Find the criterion on the triplet $(L, G, H)$ such that the action of $\mathrm{L}$ on $G / H$ is proper.

(2) Find the criterion on the triplet $(L, G, H)$ such that the double coset $L \backslash G / H$ is compact in the quotient topology.

There is a complete answer to problem 2.3 in terms of Lie algebras in [11] in the following cases:

(i) Problem 2.3(1) when $G$ is reductive (see [10]; [11], Section 2)

(ii) Problem 2.3(2) when the groups $G, H, L$ are real reductive (see [7];[11], Section 5)

Inspired by [8] Lipsman has discussed Problem 2.3 in the case where $G$ is a simply connected nilpotent Lie group. This case is considered as an opposite extremal to the semisimple case. We will give an answer to Problem 2.3(1) in a special case where $G$ is a simply connected nilpotent Lie group. 
Now we introduce some notations that are useful for a further study of problem $2.3(1)$.

\subsection{Property (CI)}

If a discrete group $\Gamma$ acts on $X$ properly discontinuously, then every isotropy subgroup is finite and every $\Gamma$ orbit is closed ([11], Lemma 2.3). The latter condition corresponds to the fact that each point is closed in the quotient topology of $\Gamma \backslash X$. In general, the converse implication does not hold (see [11], Example 2.8 for a counter example).

Kobayashi has singled out an intermediate property in a continuous setting. In fact, let $H, L$ be closed subgroups of a locally compact topological group $G$. If $L$ acts properly on $G / H$, then any $L$-orbit $L g H \cong L / L \cap g H g^{-1} \subset G / H$ is a closed subset, and each isotropy subgroup $L \cap g \mathrm{Hg}^{-1}$ is compact. In general, this condition is not sufficient for the properness of the $L$-action (see [11], Example 2.9 for a counter example). However, we pick up the second condition because of its simplicity:

Definition 2.4. ([8,11]) Suppose that $H$ and $L$ are subsets of a locally compact topological group $G$. We say that the pair $(L ; H)$ has the property $(C I)$ in $G$ if and only if $L \cap g g^{-1}$ is compact for any $g \in G$. Here $(C I)$ stands for that action of $L$ has a compact isotropy subgroup $L \cap g \mathrm{Hg}^{-1}$ at each point $g H \in G / H$, or stands for that $L$ and $g \mathrm{Hg}^{-1}$ has a compact intersection $(g \in G)$ (see also [16]).

\section{Conjecture of Lipsman}

Now we turn our attention to proper actions for nilpotent homogeneous spaces. Inspired by the results in reductive cases proved by Kobayashi [7,8], Lipsman proposed a conjecture for nilpotent groups:

Conjecture 3.1. [16] Conjecture 4.1(a)) Suppose $=N \ltimes V$, where $N=N_{k+1}(\mathbb{R})$, the group of $(k+1) \times(k+1)$ upper triangular matrices and $V=\mathbb{R}^{k+1}$. Then any connected Lie subgroup $L$ of $G$ which acts on $G / N$ with the $C I$ property, acts properly.

We note that in this case $\boldsymbol{g}=\operatorname{Lie}(G)$ is nilpotent and the following general lemma simplies the $(\mathrm{CI})$ property in the nilpotent situation.

Here, we remark that, $G$ is isomorphic to, $N^{\prime}=N_{k+2}(\mathbb{R})$

Lemma 3.2. Let $G$ be a connected, simply connected nilpotent group and let $L$ and $N$ be connected subgroups of $G$.Then the following three conditions are equivalent:

(i) $\quad(G, L, N)$ satisfies the (CI) property.

(ii) $L \cap g N g^{-1}=\{e\}$ for any $g \in G$

(iii) $\quad \boldsymbol{l} \cap \operatorname{Ad} g(\boldsymbol{n})=\{0\}$ for any $g \in G, \boldsymbol{n}=\operatorname{Lie}(N)$.

Before proving Lemma 3.2, we recall some basic results on nilpotent groups: 
Fact 3. 3. ([4], see also [5], Theorem XII.2.2,p.137). Every connected subgroup of a simply connected, solvable Lie group $G$ is closed and simply connected.

Fact 3. 4. ([5], Theorem 2.3). A compact subgroup of a simply connected, solvable Lie group is trivial.

Fact 3.5. ([20], Lemma 2.4,p.31). If $U_{1}$ and $U_{2}$ are connected subgroups of a simply connected, nilpotent Lie group $G$, then the intersection $U_{1} \cap U_{2}$ is also connected.

\section{Proof of Lemma 3.2:}

$i) \Rightarrow i i)$ : Suppose $(G, L, N)$ has the (CI) property. Then $L \cap g N g^{-1}$ is compact for any $g \in G$.

Then $L \cap g N g^{-1}=\{e\}$ by Fact 3.4.

ii) $\Rightarrow$ iii) : Obvious.

iii) $\Rightarrow$ ii) : It follows from Fact 3.5 that $L \cap g N g^{-1}$ is connected. Therefore $L \cap$ $g N g^{-1}=\{e\}$ if its Lie Algebra $\boldsymbol{l} \cap \operatorname{Adg}(\boldsymbol{n})=\{0\}$

$i i) \Rightarrow i$ ): Obvious from the defininition of the (CI) property.

\section{Statement of the Main Theorem}

Here, we are ready to state our main result of this paper.

Theorem 4. 1. For $A \in M(k ; \mathbb{R})$, we define an abelian Lie subalgebra $\boldsymbol{l}(A)$ of $g$ by

$$
\boldsymbol{l}(A)=\left\{\left(\begin{array}{ccc}
0 & A x & x \\
0 & 0 & 0 \\
0 & 0 & 0
\end{array}\right) \mid x \in \mathbb{R}^{k}\right\}
$$

We denote by $L(A)$ the connected Lie group with Lie algebra $\boldsymbol{l}(A)$. Then the following three conditions are equivalent:

(i) $L(A)$ acts properly on $G / N$

(ii) $L(A)$ acts on $G / N$ with the $C I$ property.

(iii) Any eigenvalue of the matrix $A$ lies in $\mathbb{C} \backslash \mathbb{R}^{\times}$

We put,

$\mathbb{V}:=\{A \in M(\mathrm{k}, \mathbb{R}): L(A)$ acts properly on $G / N\}$.

Corollary 4.2 If $k$ is odd, the $\mathbb{V}$ does not contain a non-empty open set of $M(k ; \mathbb{R})$.

Proof. We recall that the characteristic polynomial $f_{A}(\lambda)=\operatorname{det}(\lambda \mathrm{I}-\mathrm{A})$ is a polynomial of degree $k$ with real coefficient. If $k$ is odd then we have 


$$
\lim _{\lambda \rightarrow-\infty} f_{A}(\lambda)=-\infty, \quad \lim _{\lambda \rightarrow+\infty} f_{A}(\lambda)=\infty .
$$

Hence there is at least one real eigenvalue of $A$. Therefore, the condition (iii) is satisfied only if $\mathbb{0}$ is the eigenvalue of $A$, i.e. $\operatorname{det} A=0$.

Since eigenvalues of matrices vary continuously when we purturb the matrices, the variety $\{A \in M(k, \mathbb{R}): \operatorname{det} A=0\}$ does not contain a non-empty open subset of $M(k, \mathbb{R})$.

Thus we have proved the corollary.

Corollary 4.3. If $k$ is even, then $\mathbb{V}$ contains a non-empty open set of $M(k ; \mathbb{R})$.

Proof. Take $A \in M(k, \mathbb{R})$ to be $A=\left(\begin{array}{cc}0 & -I_{\frac{k}{2}} \\ I_{\frac{k}{2}} & 0\end{array}\right)$. Then the eigenvalues of $A= \pm i$. Since the eigenvalues of $A$ vary continuously when we purturb $A$, there exists an open subset $U$ of $A$ in $M(k ; \mathbb{R})$ such that the eigenvalues of $B$ are contained in the open set $\mathbb{C} \backslash \mathbb{R}$ for any $B \in U$.

Thus Corollary 4.3 is proved.

Remark 4.4. If $k=2$, then the matrix $\left(\begin{array}{ll}a & b \\ c & d\end{array}\right)$ has no real eigenvalue if and only if

$\lambda^{2}-(a+d) \lambda+(a d-b c)>0$ for any $\lambda \in \mathbb{R}$,

That is, the discriminant

$(a+d)^{2}-4(a d-b c)<0$,

namely, $\quad(a-d)^{2}+4 b c<0$.

Now, let

$\mathbb{V}_{0}:=\left\{A \in M_{2}(\mathbb{R}): A\right.$ has only 0 real eigenvalue $\}$

$\mathbb{V}_{1}:=\left\{A \in M_{2}(\mathbb{R}): A\right.$ has no real eigenvalue $\}$

That is, $\mathbb{V}_{0}, \mathbb{V}_{1}$ can be defined as,

$$
\begin{aligned}
& \mathbb{V}_{0}=\left\{\left(\begin{array}{ll}
a & b \\
c & d
\end{array}\right):(a-d)^{2}+4 b c<0\right\} \\
& \mathbb{V}_{1}=\left\{\left(\begin{array}{ll}
a & b \\
c & d
\end{array}\right):(a+d)=0 \text { and } a d-b c=0\right\}
\end{aligned}
$$

Therefore, we can take $\mathbb{V}$ in Corollary 4.3 such as $\mathbb{V}=\mathbb{V}_{0} \cup \mathbb{V}_{1}$.

This case $(k=2)$ was previously obtained by Lipsman (see [16], (4.5)).

Now we need a (slight) generalization of Lemma 4.2 [16] ( $k=2$ case) to a higher dimensional case.

Lemma 4.5. We denote the variety in $\boldsymbol{g}$ by,

$$
\mathfrak{B}=\bigcup_{\mathrm{u} \in \mathrm{V}} \operatorname{Ad} \mathrm{u}(\boldsymbol{n})
$$


Then we have,

$$
\begin{aligned}
\mathfrak{B} & =\{W-[W, X]: W \in \boldsymbol{n}, X \in \mathrm{v}\} \\
& =\left\{\sum_{1 \leq i<j \leq k+1}\left(c_{i j} E_{i j}\right)-\left(c_{12} y_{2}+c_{13} y_{3}+\cdots+c_{1, k+1} y_{k+1}\right) E_{1, k+2}-\left(c_{23} y_{3}+\cdots\right.\right. \\
& \left.\left.+c_{2, k+1} y_{k+1}\right) E_{2, k+2}-\cdots-\left(c_{k, k+1} y_{k+1}\right) E_{k, k+2}: c_{i j}, y_{i} \in \mathbb{R}\right\}
\end{aligned}
$$

Proof. If $u=e^{X} \in V(X \in \boldsymbol{v})$ and $W \in \boldsymbol{n}=\operatorname{Lie}(N)$, then we have

$$
\begin{aligned}
& \operatorname{Ad}(u) W=\operatorname{Ad}\left(e^{X}\right) W=e^{\operatorname{ad} X} W \\
& (*) \quad=W+[X, W]+\frac{1}{2}[X,[X, W]]+\cdots
\end{aligned}
$$

Because

$$
\begin{aligned}
& {[X, W] \in[\boldsymbol{v}, \boldsymbol{n}] \subset \boldsymbol{v} \text { and }[\mathrm{X},[X, W]] \in[\boldsymbol{v}, \boldsymbol{v}]=\{0\} \text {, we have (from (*)) }} \\
& W+[X, W]+\frac{1}{2}[X,[X, W]]+\cdots \\
& =\mathrm{W}+[\mathrm{X}, \mathrm{W}]=\mathrm{W}-[\mathrm{W}, \mathrm{X}] .
\end{aligned}
$$

Hence the first equality is proved. Now if we write

$$
\begin{aligned}
& W=\sum_{1 \leq i<j \leq k+1} c_{i j} E_{i j} \in \boldsymbol{n} \text { and } X=\sum_{i=1}^{k+1} y_{l} E_{l, k+2} \in \boldsymbol{v} \text {, then, } \\
& {[W, X]=\sum_{i, j, l} c_{i j} y_{l}\left[E_{i j}, E_{l, k+2}\right]=\sum_{1 \leq i<j \leq k+1} c_{i j} y_{j} E_{i, k+2}}
\end{aligned}
$$

This is because,

$$
\left[E_{i j}, E_{l, k+2}\right]= \begin{cases}0 & \text { if } j \neq l \\ E_{i, k+2} & \text { if } j=l\end{cases}
$$

where $1 \leq i<j \leq k+1$.

Thus the second equality is also proved

Proposition 4.6. SupposeL $(A)$ acts on $G / N$ with the property CI. Then for any non-zero element $W+X \in \boldsymbol{l}(A)$ with $W \in \boldsymbol{n}, X \in \boldsymbol{v}$, we have $X \notin \operatorname{Range}\left(\operatorname{ad}_{\boldsymbol{v}} W\right)$.

Proof. It follows from Lemma 3.2 and from the definition of $\mathfrak{B}$ that $\boldsymbol{l}(A) \cap \mathfrak{B}=\{0\}$. Therefore, if $\{0\} \neq W+X \in \boldsymbol{l}(A)$, then $W+X \notin \mathfrak{B}$, which implies $X \notin$ Range $\left(\operatorname{ad}_{v} W\right)$ by Lemma 4.5 .

\section{Proof of the main theorem}

In this section we give a proof of the precise version (that is, the three equivalent conditions) in Theorem 4.1. For this purpose we need to establish the following two lemmas: 
Lemma 5.1. If the matrix $A=\left(a_{i j}\right)_{1 \leq i, j \leq k}$ has no real eigenvalue except for 0 then $L(A)$ acts on $G / N$ properly.

Remark 5. 2. The converse is also true. It will be proved after Lemma 5.3.

Proof of Lemma 5.1. To establish that $L(A)$ acts properly on $G / N$, we shall prove that $L(A) \cap S N S^{-1}$ is compact for any compact set $S \subset V$. Because $\boldsymbol{l}(A)$ is abelian, each $\ell \in L(A)$ is of the form

$$
\begin{aligned}
& \ell=\exp \left(\sum_{j=1}^{k} \lambda_{j} T_{j}\right) \\
& =\exp \left(\sum_{j=1}^{k} \sum_{i=1}^{k} \lambda_{j} a_{i j} E_{i, k+1}\right) \exp \left(\sum_{j=1}^{k} \lambda_{j} E_{j, k+2}\right) \in N_{k+1}(\mathbb{R}) \ltimes \mathrm{V}
\end{aligned}
$$

for some $\lambda_{1}, \ldots, \lambda_{k} \in \mathbb{R}$ and where for $1 \leq j \leq k$ we put

$$
T_{j}=\sum_{i=1}^{k} \lambda_{j} a_{i j} E_{i, k+1}+E_{j, k+2}
$$

It is convenient to define,

$$
\begin{aligned}
& s(x):=\exp \sum_{j=1}^{k+1} \mathrm{x}_{\mathrm{j}} \mathrm{E}_{j, k+2}=\left(\begin{array}{ccccc}
1 & 1 & \cdots & 0 & \mathrm{x}_{1} \\
& & \ddots & \vdots & \vdots \\
0 & \ddots & \ddots & 0 & \mathrm{x}_{k} \\
& & & 1 & \mathrm{x}_{k+1} \\
\text { for } x=\left(x_{1}, \cdots, x_{k+1}\right) \in \mathbb{R}^{k+1} & & & & 1
\end{array}\right)
\end{aligned}
$$

Since $S$ is compact, $S$ can be written as

$$
S=\{\mathrm{s}(\mathrm{x}): x \in B\}
$$

Here $B$ is a compact subset of $\mathbb{R}^{k+1}$.

Let us compute $s(y) n s(x)[s(x) n s(y)]$ for $s(y), s(x)^{-1} \in S$ and for

Suppose we have

$$
\boldsymbol{n}=\left(\begin{array}{ccccc}
1 & \mathrm{~b}_{12} & \cdots & \mathrm{b}_{1, k+1} & 0 \\
& & \ddots & \mathrm{b}_{k, k+1} & 0 \\
0 & \ddots & \ddots & 1 & 0 \\
& & & & 1
\end{array}\right) \in \mathrm{N}_{k+1}(\mathbb{R})
$$

$$
s(y) n s(x)=n^{\prime} s(z),
$$

where $\mathrm{n}^{\prime} \in N_{k+1}(\mathbb{R})$ and $s(z) \in V$. Then we obtain, 


$$
\left\{\begin{array}{c}
\mathrm{n}^{\prime}=n \\
z_{i}=x_{i}+y_{i}(-)+b_{i, k+1} y_{k+1} \bmod \left\langle b_{i j} ; 1 \leq i<j \leq k\right\rangle \\
z_{k+1}=x_{k+1}+y_{k+1}
\end{array}\right.
$$

In particular, if $s(y) n s(x) \in L(A)$ then by comparing with (5.1), we have

$$
\begin{aligned}
& b_{i j}=0 \text { for } 1 \leq i<j \leq k \\
& x_{k+1}+y_{k+1}=0 \\
& \overrightarrow{\mathrm{x}}+\overrightarrow{\mathrm{y}}=\vec{\lambda}-\left(\begin{array}{c}
\mathrm{b}_{1, \mathrm{k}+1} \\
\mathrm{~b}_{2, \mathrm{k}+1} \\
\vdots \\
\mathrm{b}_{\mathrm{k}, \mathrm{k}+1}
\end{array}\right) \mathrm{y}_{\mathrm{k}+1} \\
& \left(\begin{array}{c}
b_{1, k+1} \\
b_{2, k+1} \\
\vdots \\
b_{k, k+1}
\end{array}\right)=A \vec{\lambda}
\end{aligned}
$$

where $A=\left(a_{\mathrm{ij}}\right)_{1 \leq i, j \leq k}$ and

$$
\vec{x}=\left(\begin{array}{c}
x_{1} \\
x_{1} \\
\vdots \\
x_{k}
\end{array}\right), \quad \vec{y}=\left(\begin{array}{c}
y_{1} \\
y_{1} \\
\vdots \\
y_{k}
\end{array}\right), \quad \lambda=\left(\begin{array}{c}
\lambda_{1} \\
\lambda_{1} \\
\vdots \\
\lambda_{k}
\end{array}\right)
$$

From equations (5.2), (5.3), (5.4), (5.5), we obtain a matrix equation,

$$
\vec{x}+\vec{y}=\left(I_{k}-y_{k+1} A\right) \vec{\lambda}
$$

From our assumption that $A$ has no real eigenvalue except for 0 , it follows that

$$
\operatorname{det}\left(I_{k}-y_{k+1} A\right) \neq 0 \quad \text { (for any } y_{k+1} \in \mathbb{R}^{\times} \text {). }
$$

Suppose $y_{k+1}$ is contained in a compact set $I$ of $\mathbb{R}^{\times}$. Then there exists a constant $\delta>0$ such that,

$$
\left|\operatorname{det}\left(I_{k}+y_{k+1} A\right)\right|>\delta \text { for any } y_{k+1} \in \mathbb{R}^{\times} \text {. }
$$

Then there exists a constant $M>0$ such that

$\left|(\mathrm{C})_{\mathrm{ij}}\right| \leq M$ for any $y_{k+1} \in \mathbb{R}^{\times}$. and any $1 \leq i, j \leq k$

Here $C_{i j}$ denotes the $(i, j)$ th element of the matrix $\mathrm{C}=\left(I_{k}+y_{k+1} A\right)^{-1}$ 
Because $\vec{x}, \vec{y}$ are elements in the bounded set $B \subset \mathbb{R}^{k+1}$, the solution $\vec{\lambda}$ of the equation (5.6) is bounded. In view of the definition of $\ln (5.1), L(A) \cap S N S^{-1}$ is compact. Therefore, we have proved that the action of $L(A)$ is proper.

Lemma 5.3. If the action of $L(A)$ on $G / N$ has the (CI) property, then the matrix $A \in$ $M_{k}(\mathbb{R})$ has no non-zero real eigenvalue.

Proof. Any element of $\boldsymbol{l}(A)$ is of the form

$$
\sum_{j=1}^{k} \lambda_{j} T_{j} \quad\left(\lambda_{1}, \lambda_{2}, \ldots, \lambda_{k} \in \mathbb{R}\right) .
$$

By defnition 4.1, ·

$$
\sum_{j=1}^{k} \lambda_{j} T_{j}=\sum_{j=1}^{k} \sum_{i=1}^{k} \lambda_{j} a_{i j} E_{i, k+1}+\sum_{j=1}^{k} \lambda_{j} E_{j, k+2} \in \boldsymbol{n}+\boldsymbol{v}
$$

If $\left(\lambda_{1}, \lambda_{2}, \cdots, \lambda_{k}\right) \neq(0,0, \ldots, 0)$, then by Proposition 4.6 we have,

$$
\sum_{j=1}^{k} \lambda_{j} E_{j, k+2} \notin \operatorname{Range}\left(\operatorname{ad}_{\boldsymbol{v}}\left(\sum_{j=1}^{k} \sum_{i=1}^{k} \lambda_{j} a_{i j} E_{i, k+1}\right)\right)
$$

In order to compute the right side of (5.7), we use:

$$
\left[\left(\sum_{j=1}^{k} \sum_{i=1}^{k} \lambda_{j} a_{i j} E_{i, k+1}\right),\left(\sum_{j=1}^{k+1} x_{j} E_{j, k+2}\right)\right]=x_{k+1}\left(\sum_{j=1}^{k} \sum_{i=1}^{k} \lambda_{j} a_{i j} E_{i, k+2}\right)
$$

for $u=\sum_{j=1}^{k+1} x_{j} E_{j, k+2}$

The condition (5.7) implies that the system of equations for $\lambda_{1}, \lambda_{2}, \ldots, \lambda_{k}$ :

$$
\left\{\begin{array}{c}
\lambda_{1}=x_{k+1} \sum_{j=1}^{k} \lambda_{j} a_{1 j} \\
\lambda_{2}=x_{k+1} \sum_{j=1}^{k} \lambda_{j} a_{2 j} \\
\vdots \\
\lambda_{k}=x_{k+1} \sum_{j=1}^{k} \lambda_{j} a_{k j}
\end{array}\right.
$$

has no solution except for $\left(\lambda_{1}, \lambda_{2}, \cdots, \lambda_{k}\right)=(0,0, \cdots, 0)$ for each fixed $x_{1}, x_{2} \cdots, x_{k+1}$. In a matrix form, (5.8) is expressed as

$$
\left(I_{k}-x_{k+1} A\right) \vec{\lambda}=0 .
$$

Therefore, $\operatorname{det}\left(I_{k}-x_{k+1} A\right) \neq 0$ for any $x_{1}, \cdots, x_{k+1} \in \mathbb{R}$. This is equivalent to the condition that $A$ has no non-zero real eigenvalue.

Therefore we have proved Lemma 5.3.

Thus we have completed the proof of Theorem 4.1. In particular Lipsman's conjecture holds for the triplet $(L(A), G, N)$. 


\section{Acknowledgements}

This manuscript is written when the author was visiting the Department of Mathematics at the University Paul Verlaine-Metz, France. So the author is grateful to her host Professor Salah Mehdi for his hospitality and helpful conversations during her stay in Metz, France. Thanks are also due to Professor Toshiyuki Kobayashi for his much valuable advice and guidance in the preparation of this manuscript.

\section{References}

1. A. Baklouti and F. Khlif, Geom. Dedicata 146, 117 (2010). http://dx.doi.org/10.1007/s10711-009-9429-3

2. A. Baklouti and F. Khlif, Int. J. Math. 16(9), 941 (2005). http://dx.doi.org/10.1142/S0129167X0500317X

3. E. Calabi and L. Markus, Ann. Math. 75, 63 (1962). http://dx.doi.org/10.2307/1970419

4. C. Chevalley, Ann. of Math. 42, 668 (1941).

5. G. Hochschild, The structure of Lie groups (San Francisco: Holden-day, 1965).

6. F. Kassel and T. Kobayashi, C. R. Math. Acad. Sci. Paris 349(1-2), 29 (2011).

7. T. Kobayashi, Math. Ann. 285, 249 (1989). http://dx.doi.org/10.1007/BF01443517

8. T. Kobayashi, Discontinuous groups acting on homogeneous spaces of reductive type, Proceedings of the Conference on Representation Theory of Lie Groups and Lie Algebras held in 1990 August-September at Fuji-Kawaguchiko (ICM-90 Satellite Conference) (World Scientific, Singapore/New Jersey/London, 1992) pp. 59-75.

9. T. Kobayashi, J. Geometry and Physics 12, 133 (1993). http://dx.doi.org/10.1016/0393-0440(93)90011-3

10. T. Kobayashi, J. Lie Theory 6, 147 (1996).

11. T. Kobayashi, Discontinuous Groups and Clifford-Klein Forms of Pseudo-Riemannian Homogeneous Manifold, in 'Algebraic and analytic methods in representation theory' edited by B. Ørsted and H. Schlichtkrull (Academic Press, 17, 99, 1996).

12. T. Kobayashi, Math. Ann. 310, 395 (1998). http://dx.doi.org/10.1007/s002080050153

13. T. Kobayashi, On discontinuous group actions on non-Riemannian homogeneous spaces, Sugaku Expositions 22 (2009), (Amer. Math. Soc. 1-19; a translation by Miles Reid of the original article in Japanese).

14. T. Kobayashi, Mathematische Arbeitstagung, MPIM2009-40n, 15 (2009).

15. R. S. Kulkarni, Advances in Math. 40, 10 (1981). http://dx.doi.org/10.1016/0001-8708(81)90031-1

16. R. Lipsman, J. Lie Theory. 5, 25 (1995).

17. S. Nasrin, Tokyo J. Math. Vol. 24(2), 535 (2001).

18. T. Okuda, Proc. Japan Acad. Ser. A Math. Sci. 87, 35 (2011). http://dx.doi.org/10.3792/pjaa.87.35

19. R. S. Palais, Ann. of Math. 73, 295 (1961). http://dx.doi.org/10.2307/1970335

20. M. S. Raghunathan, Discrete Subgroups of Semisimple Lie Groups (Berlin Heidelberg New York: Springer, 1972).

21. A. Weil, Ann. of Math. 80, 149 (1964). http://dx.doi.org/10.2307/1970495

22. J. A.Wolf, Ann. of Math. 75, 77 (1962). http://dx.doi.org/10.2307/1970420

23. T. Yoshino, Int. J. Math. 16, 561(2005). http://dx.doi.org/10.1142/S0129167X05002965

24. T. Yoshino, Int. J. Math. 85, 887 (2007). http://dx.doi.org/10.1142/S0129167X07004370 